UVX 2012, $02002(2013)$

DOI: $10.1051 /$ uvx/201302002

(C) Owned by the authors, published by EDP Sciences, 2013

\title{
Génération d'harmoniques d'ordre élevé en régime de forte focalisation à $100 \mathrm{kHz}$
}

\author{
A. Cabasse, G. Machinet, C. Hazera, S. Petit, E. Cormier \\ et E. Constant \\ Univ. Bordeaux, CEA, CNRS, CELIA, UMR 5107, 33400 Talence, France
}

\begin{abstract}
Résumé. De nombreuses applications nécessitent des sources XUV à haut taux de répétition avec un important nombre de photons XUV par impulsion. Potentiellement ces sources peuvent être obtenues par génération d'harmoniques d'ordre élevé avec un laser fondamental à haute cadence. Cependant, la faible énergie et la longue durée des impulsions délivrées par ces systèmes laser exigent de focaliser fortement le faisceau fondamental afin d'atteindre des éclairements adéquats pour la génération d'harmoniques $\left(\sim 10^{14} \mathrm{~W} / \mathrm{cm}^{2}\right)$. Ceci s'avère généralement défavorable pour l'efficacité du processus qu'il convient d'optimiser. Dans ce travail, une étude numérique permet de définir des conditions générales d'optimisation de la génération d'harmoniques en forte focalisation et nous observons la signature d'un accord de phase parfait. Ces conditions sont reproduites expérimentalement et nous obtenons une optimisation de l'émission harmonique avec un nombre de photons XUV (harmoniques d'ordres 15-17 et 19) émis dans du Xe supérieur à $10^{12} \mathrm{~s}^{-1}$ à la cadence de $100 \mathrm{kHz}$.
\end{abstract}

\section{INTRODUCTION}

La génération d'harmoniques d'ordre élevé (HHG) est un phénomène bien connu permettant l'émission d'un rayonnement XUV cohérent ultra-court. Historiquement, les lasers utilisés pour générer des harmoniques délivraient des impulsions longues $(\sim$ ps $)[1,2]$. Le faisceau fondamental devait donc être fortement focalisé dans le milieu gazeux afin d'atteindre les éclairements nécessaires à la génération d'harmoniques $\left(>10^{13} \mathrm{~W} / \mathrm{cm}^{2}\right)$. Le développement de sources laser intenses et ultra-courtes (principalement les sources laser Titane saphir) ont cependant permis de réduire cette focalisation et de travailler en configuration dite de «longue focale », ce qui accroît l'efficacité de génération.

La tendance actuelle est d'utiliser des systèmes laser de haute cadence et de forte puissance moyenne [3,4]. La faible énergie associée à ces systèmes nécessite néanmoins de revenir à la géométrie initiale de forte focalisation, limitant ainsi le rendement du processus. À ce jour, différents groupes ont généré des harmoniques à très haute cadence dans une telle géométrie. Boullet et al. ont utilisé un laser à fibre CPA (Chirped Pulse Amplification) à une cadence pouvant aller de $100 \mathrm{kHz}$ à $1 \mathrm{MHz}$. En considérant une efficacité de conversion de $10^{-6}$, un nombre de photons XUV de $3.10^{11}$ photons/s a été estimé [5]. Un système laser similaire a permis de mesurer une efficacité de conversion de $1,2.10^{-9}$. Une technique de post-compression a été implémentée sur ce système conduisant à une augmentation de l'efficacité de conversion $\left(1,2.10^{-7}\right.$ ce qui correspond à un nombre de photons XUV émis de $7,9.10^{11}$ photons/s) [6]. En utilisant un système laser à $100 \mathrm{kHz}$ délivrant des impulsions de seulement quelques $\mu \mathrm{J}$ d'énergie centrées à $800 \mathrm{~nm}$, Heyl et al. ont généré des harmoniques dans différents gaz avec un flux XUV de $1.10^{9}$ photons/s (efficacité de $10^{-8}$ ) [7].

Dans ce travail, une étude numérique permet de définir des conditions permettant d'optimiser l'efficacité de la génération d'harmoniques dans un régime de forte focalisation et en utilisant un système laser à très haut taux de répétition. Nous analysons l'influence de différents paramètres : le gaz (argon,

This is an Open Access article distributed under the terms of the Creative Commons Attribution License 2.0, which permits unrestricted use, distribution, and reproduction in any medium, provided the original work is properly cited. 

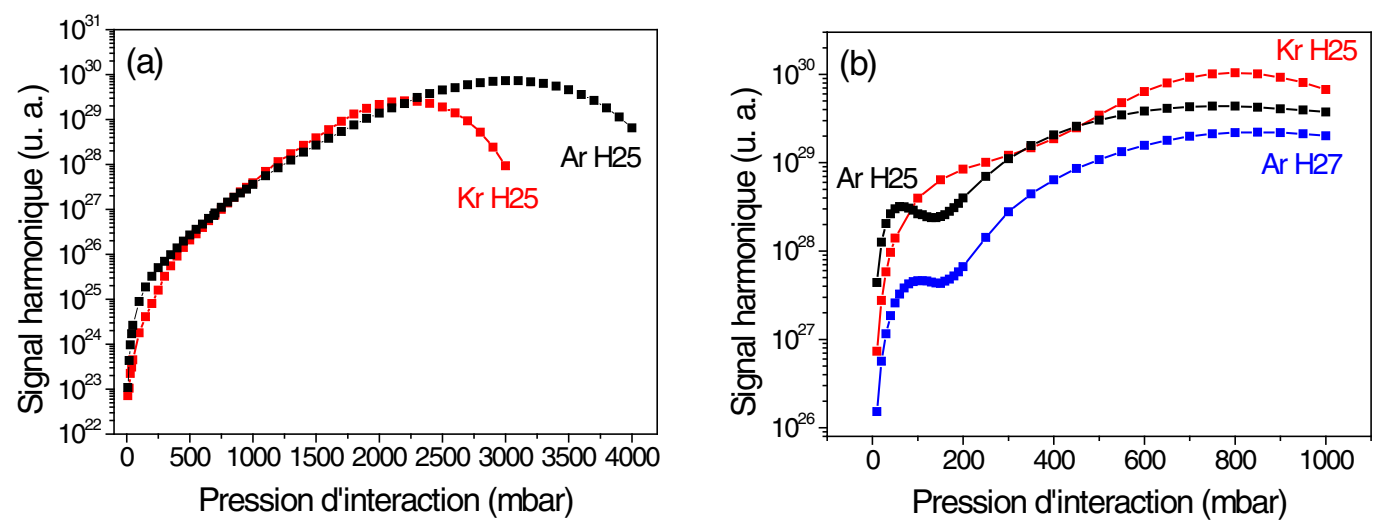

Figure 1. Signal de différents ordres harmoniques en fonction de la pression d'interaction (a) dans un jet $\left(\mathrm{L}_{\text {milieu }}=\right.$ $200 \mu \mathrm{m}$ ) et (b) dans une cellule pour différents gaz (argon, krypton).

krypton ou xénon), sa pression et la géométrie d'interaction laser/gaz (un jet fin ou une cellule). Dans certaines conditions, un accord de phase parfait ou une optimisation de l'émission harmonique sont obtenus. Ces conditions sont reproduites expérimentalement et nous mesurons un nombre de photons XUV émis (harmoniques 15,17 et 19) de plus de $10^{12}$ photons/s dans le xénon.

\section{2. ÉTUDE \& RÉSULTATS NUMÉRIQUES}

Tout d'abord, une analyse numérique est réalisée en configuration de forte focalisation. Le code de propagation 3D modélise la construction du champ harmonique émis en champ lointain en tenant compte du profil spatial du champ fondamental dans le milieu gazeux, des conditions d'accord de phase (longueur de cohérence $\mathrm{L}_{\mathrm{coh}}$ ) et de l'effet de l'absorption (longueur d'absorption $\mathrm{L}_{\mathrm{abs}}$ ). Le dipôle harmonique varie comme une loi de puissance de l'éclairement laser (ordre 1,5 lorsque l'harmonique est dans le plateau et ordre 5 au-delà de la coupure) et sa phase est proportionnelle à l'éclairement (coefficient de proportionnalité $\alpha=2.5 \mathrm{~cm}^{2} / \mathrm{W}$ ). Le signal harmonique est calculé en utilisant un éclairement au foyer de $6.10^{13} \mathrm{~W} / \mathrm{cm}^{2}$ (waist $\mathrm{w}_{0}=10 \mu \mathrm{m}$, paramètre confocal $\mathrm{b}=610 \mu \mathrm{m}$ ). Le profil temporel des impulsions centrées à $1030 \mathrm{~nm}$ est négligé ainsi que l'ionisation.

La figure 1 présente le signal XUV de différents ordres harmoniques émis dans différentes géométries caractérisées par leur longueur : un jet de gaz $\left(\mathrm{L}_{\text {milieu }}=200 \mathrm{~lm}\right)$ (Fig. 1-a) et une cellule $\left(\mathrm{L}_{\text {milieu }}=1 \mathrm{~mm}\right)($ Fig. 1-b), en fonction de la pression d'interaction.

Avec le jet, le signal augmente puis décroit au delà d'une pression optimale proche de 2 à 3 bars (variant suivant le gaz et l'ordre harmonique considéré). L'utilisation d'une cellule montre une évolution similaire avec une augmentation rapide du signal, une saturation puis une diminution. Néanmoins, avec cette géométrie, la saturation apparaît pour des pressions optimales nettement plus basses ( $~ 800$ mbar). Ces simulations montrent aussi des structures nettes à basse pression avec un maximum suivi par un minimum local qui sont explicables par les conditions d'accord de phase [8]. Le premier maximum ( $\sim 100$ mbar) apparait lorsque la longueur de cohérence, $\mathrm{L}_{\mathrm{coh}}$, devient infinie (un accord de phase parfait) à la pression ou la dispersion du gaz compense la dispersion géométrique. Le minimum ( 200 mbar) correspond à des interférences destructives entre les harmoniques générées dans la première et la seconde moitié du milieu ( $\left.\mathrm{L}_{\mathrm{coh}}=\mathrm{L}_{\text {milieu }} / 2\right)$. Enfin, autour de $800 \mathrm{mbar}$, on remarque que le signal obtenu avec la cellule est environ deux ordres de grandeur supérieur à celui obtenu avec le jet. Cependant, ils sont tous deux comparables lorsque la saturation est atteinte pour chacun des systèmes.

Cette étude numérique montre que le signal harmonique est similaire dans une cellule et dans un jet lorsque les conditions optimales sont obtenues. Néanmoins, le jet nécessite de plus fortes pressions 

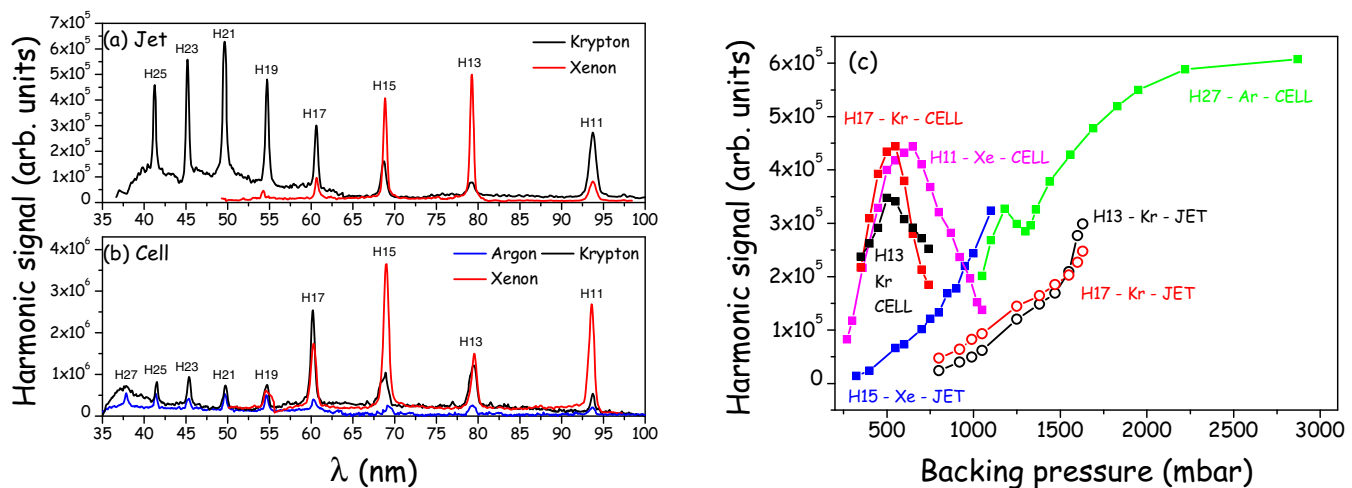

Figure 2. Spectres harmoniques obtenus à $100 \mathrm{kHz}$ avec (a) un jet, (b) une cellule. (c) Evolution du signal harmonique en fonction de la pression en amont, pour différents gaz (les unités changent d'une harmonique à l'autre).

d'interaction pour atteindre le maximum du signal harmonique et ces pressions optimales sont d'autant plus grandes que la focalisation est forte. Ceci peut s'avérer être une contrainte expérimentale forte d'autant que dans un jet, la pression d'interaction est environ dix fois plus faible que la pression en amont. Dans une cellule, ces deux pressions sont équivalentes. Nous pouvons donc nous attendre, en utilisant expérimentalement une cellule, à une augmentation du signal XUV.

\section{3. ÉTUDE \& RÉSULTATS EXPÉRIMENTAUX}

Le modèle numérique a ensuite été testé expérimentalement en utilisant un système femtoseconde CPA. A la cadence de $100 \mathrm{kHz}$ et à $1030 \mathrm{~nm}$, le système laser délivre des impulsions de $500 \mathrm{fs}$ de durée et de $10 \mathrm{~W}$ de puissance moyenne ce qui correspond à une énergie par impulsion de $100 \mu \mathrm{J}$. Les harmoniques d'ordre élevé sont générées en focalisant le faisceau fondamental avec une lentille de $100 \mathrm{~mm}\left(w_{0}=10 \mu \mathrm{m}\right)$ dans une première chambre à vide. L'éclairement au foyer est estimée à $6.10^{13} \mathrm{~W} / \mathrm{cm}^{2}$. Suite à l'étude numérique, différentes géométries d'interaction laser/gaz ont été étudiées pour optimiser l'efficacité de conversion. Le flux XUV émis est caractérisé par un spectromètre XUV composé d'une fente, d'un réseau de diffraction $\left(600 \mathrm{tr} / \mathrm{mm}, \mathrm{i}=87^{\circ}\right)$ et d'un détecteur, composé de galettes de micro-canaux, d'un écran phosphore et d'une caméra CCD.

Des harmoniques ont été générées dans un jet effusif $\left(\mathrm{L}_{\text {milieu }}=200 \mu \mathrm{m}\right)$, dans du krypton $(\mathrm{H} 11$ à H25) et du xénon (H13 à H19) (figure 2-a). L'utilisation d'une cellule ( $\mathrm{L}_{\text {milieu }}=1 \mathrm{~mm}$ ) nous a ensuite permis d'observer des harmoniques dans du krypton (H11 à H27), du xénon (H11 à H19) et de l'argon (H11 à H27) (figure 2-b). L'évolution du signal harmonique intégré en fonction de la pression en amont montre une augmentation régulière pour le jet mais aucun optimum n'est atteint, lié à une limitation du pompage (pompe turbo de 5001/s) (figure 2-c). Avec la cellule, nous observons une saturation du signal pour une pression optimale. À basse pression, les structures liées aux conditions d'accord de phase sont bien observées dans le cas de l'argon.

Enfin, un filtre aluminium (épaisseur $=200 \mathrm{~nm}$ ) et une photodiode couplée un pico-ampèremètre ont permis de mesurer le flux XUV émis, en estimant à 50\% la transmission du filtre. Le tableau 1 donne les résultats obtenus pour le jet et pour la cellule, dans les différents gaz étudiés.

L'utilisation d'une cellule a conduit à une nette amélioration du flux XUV, avec une estimation du flux émis dans le xénon à plus de $10^{12}$ photons/s. Les harmoniques d'ordre élevé ont pu être détectées et étudiées avec ce spectromètre XUV, mais ce flux ne peut cependant pas être directement utilisé pour des expériences résolues en temps. Le spectromètre a donc été amélioré pour pouvoir réaliser prochainement de telles expériences et refocaliser le faisceau XUV en le contrôlant (spectralement ou temporellement). 
Tableau 1. Mesure du nombre de photons XUV émis (ordres harmoniques $>15$ ) et efficacité de conversion.

\begin{tabular}{|c|c|c|c|}
\cline { 2 - 4 } \multicolumn{1}{c|}{ JET } & GAS & $\mathrm{N}_{\mathrm{ph}}\left(\mathrm{s}^{-1}\right)(>\mathrm{H} 15)$ & $\eta$ (par harmonique) \\
\hline \multirow{3}{*}{ CELLULE } & Krypton & $8,5.10^{10}$ & $5,6.10^{-9}$ \\
\cline { 2 - 4 } & Xénon & $3,5.10^{11}$ & $3,2.10^{-8}$ \\
\cline { 2 - 4 } & Argon & $1.10^{11}$ & $7.10^{-9}$ \\
\cline { 2 - 4 } & Krypton & $4.10^{11}$ & $2,8.10^{-8}$ \\
\cline { 2 - 4 } & Xénon & $\mathbf{1 , 2 . 1 0}^{12}$ & $\mathbf{1 , 6 . 1 0}^{-7}$ \\
\hline
\end{tabular}

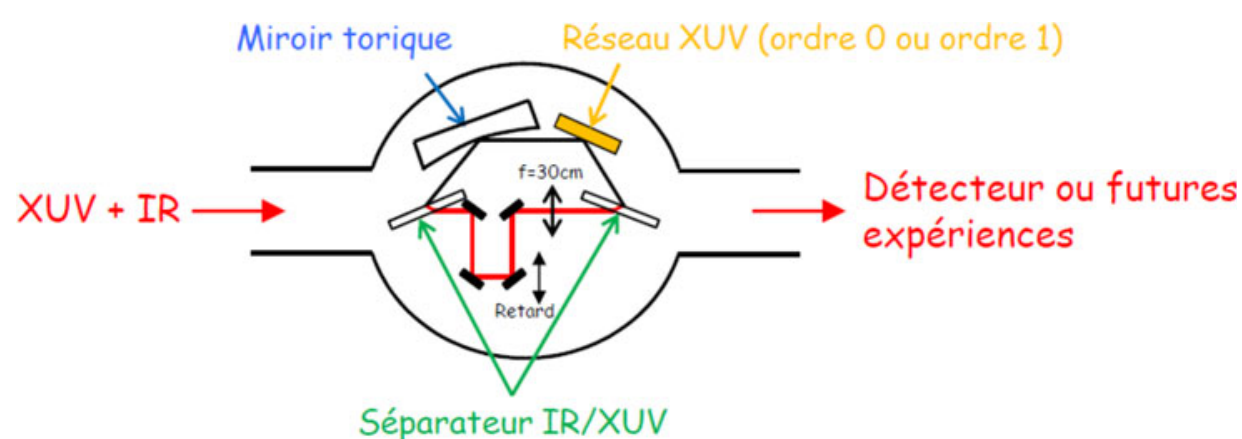

Figure 3. Spectromètre XUV avec contrôle de l'IR.

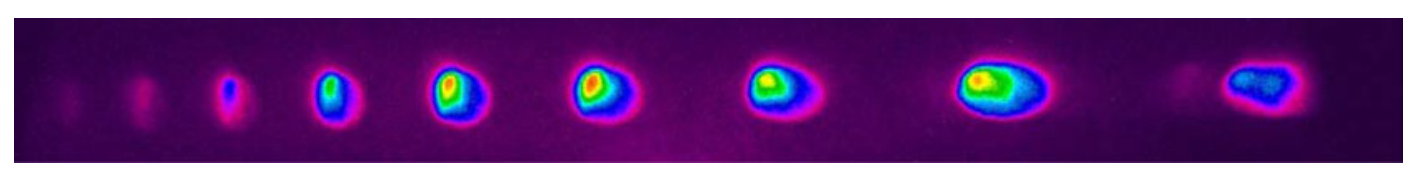

Figure 4. Spectre d'harmoniques générées dans du krypton à la pression de 500 mbar (H13 à H29).

\section{4. ÉVOLUTION DU SYSTÈME}

Un nouveau spectromètre XUV a été conçu pour réaliser de futures expériences résolues en temps avec une résolution temporelle femtoseconde (figure 3) et compatible avec la forte charge thermique de ce type de laser. Le faisceau XUV est tout d'abord réfléchi par un premier séparateur qui transmet la majeure partie du faisceau fondamental IR copropageant. Après cette réflexion, le faisceau XUV est réfléchi par un miroir torique ( $\mathrm{f}=30 \mathrm{~cm}$ qui focalise le faisceau à $80 \mathrm{~cm}$ du miroir) vers un réseau utilisé soit dans l'ordre zéro (pour une résolution temporelle) soit dans l'ordre 1 (pour une résolution spectrale). Un second séparateur permet ensuite de recombiner les deux faisceaux XUV et IR qui peuvent être refocalisés au même point. Le faisceau fondamental IR passe quant à lui à travers une ligne à retard variable qui permet de contrôler finement le délai entre les impulsions XUV et IR. Une lentille de même focale que le miroir torique est installée sur le bras IR pour permettre un recouvrement des foyers IR et XUV. L'éclairement de l'IR pourra être contrôlé en plaçant un iris à diamètre variable ou une lame quart d'onde suivie d'un polariseur.

Ce nouveau système a été testé pour caractériser des harmoniques d'ordre élevé avec un second laser CPA fibré, délivrant des impulsions de 570 fs, $46 \mathrm{~W}, 460 \mu \mathrm{J}$ centrées à $1030 \mathrm{~nm}$ et à la cadence de $100 \mathrm{kHz}$. Une lentille de $200 \mathrm{~mm}$ permet de focaliser le faisceau fondamental dans une cellule de gaz. L'éclairement au foyer est estimé à $7 \times 10^{13} \mathrm{~W} / \mathrm{cm}^{2}$. L'observation d'harmoniques sur le détecteur a permis de valider ce nouveau spectromètre XUV et nous n'avons observé aucun dommage lié à la charge thermique élevée (figure 4). Nous avons mesuré à la sortie du système un flux de $5.10^{10}$ photons/s, ce 


\section{UVX 2012}

qui correspond, en prenant compte des réflexions des différentes optiques dans le spectromètre, à un nombre de photons émis dans la zone d'interaction de $2.10^{12}$ photons/s.

\section{CONCLUSIONS ET PERSPECTIVES}

Pour conclure, nous avons étudié, en faisant varier différents paramètres (gaz, pression et géométrie), l'optimisation d'une source XUV à la cadence de $100 \mathrm{kHz}$ en utilisant un système laser fibré ultra-court et de forte puissance moyenne. Nous avons pu montrer une augmentation du flux XUV en utilisant une cellule de $1 \mathrm{~mm}$ de long avec un nombre de photons émis de plus de $10^{12}$ photons/s dans le xénon. Le développement d'une nouvelle configuration expérimentale va permettre d'utiliser ces photons pour de futures expériences résolues en temps et à très haute cadence.

\section{Remerciements}

Les auteurs remercient l'Agence Nationale de la Recherche (ANR-09-BLAN-0031-01), le Conseil Régional d'Aquitaine (NASA, Femto+) et la communauté Européenne (no. 228334 JRA-ALADIN et INREX Laserlab Europe II et III ).

\section{Références}

[1] A. McPherson, G. Gibson, H. Jara, U. Johann, T. S. Luk, I. McIntyre, K. Boyer, C. K. Rhodes, J. Opt. Soc. Am. B 4, 595 (1987)

[2] M. Ferray, A. L'Huillier, K. F. Li, L. A. Lompré, G. Mainfray, C. Manus, J. Phys. B 21, L31 (1988)

[3] Lindner et al. Phys. Rev. A 68, 013814 (2003)

[4] C. M. Heyl, J. Güdde, U. Höfer, A. L'Huillier, Phys. Rev. Lett. 107, 033903 (2011)

[5] J. Boullet, Y. Zaouter, J. Limpert, S. Petit, Y. Mairesse, B. Fabre, J. Higuet, E. Mével, E. Constant, E. Cormier, Opt. Lett. 34, 1489 (2009)

[6] S. Hädrich, M. Krebs, J. Rothhardt, H. Carstens, S. Demmler, J. Limpert, A. Tünnermann, Opt. Express 19, 19374 (2011)

[7] C. M. Heyl, J. Güdde, A. L'Huillier, U. Höfer, J. Phys. B: At. Mol. Opt. Phys. 45, 074020 (2012)

[8] S. Kazamias, S. Daboussi, O. Guilbaud, K. Cassou, D. Ros, B. Cros, G. Maynard, Phys. Rev. A 83, 063405 (2011) 